\title{
Early Career Development in Academic Pediatrics of Participants in the APS-SPR Medical Student Research Program
}

\author{
WILLIAM H. SMITH, JESSICA G. ROGERS, THOMAS N. HANSEN, AND CHARLES V. SMITH
}

Department of Pediatrics, University of Washington School of Medicine, Seattle, WA 98101

\begin{abstract}
To recruit and train the next generations of pediatric clinician-scientists, the American Pediatric Society and Society for Pediatric Research initiated a program in 1991 to support medical students with interests in research and pediatrics to conduct research at institutions other than their respective medical schools. Since 1991, the American Pediatric Society-Society for Pediatric Research Medical Student Research Program (MSRP) has funded 732 of 2209 applicants from 132 US or Canadian medical schools for 8-12 wk of research under the direction of experienced investigators. PubMedattributable publications tabulated in 2001 for MSRP applicants through 2000 indicated that participants had published more actively than had nonparticipant applicants. Male nonparticipants exhibited greater publication activities than did female nonparticipants, but female and male participants published equally. Of all MSRP participants between 1991 and 1996, as of 2008, 36\% were in pediatrics, and a remarkable 29\% were in academic pediatrics. (Pediatr Res 65: 474-477, 2009)
\end{abstract}

$\mathrm{C}$ linician-scientists are important to progress in biomedical research, because clinician-investigators can provide diversities of experience and training that are particularly crucial in biomedical research needed for continued improvements in clinical care (1-3). Differences in cultural backgrounds and ethnicities also bring diversity to the problems facing health care development and delivery. Minority medical school graduates are more likely to practice in medically underserved communities than are nonminority medical graduates, and minority clinician-scientists are likely to be more active in investigating problems that affect patients who would otherwise remain underrepresented in the biomedical research enterprise (4-7).

To encourage more medical students, especially those from underrepresented racial and ethnic groups, to consider research in their career paths, increases in exposure to and training in research are needed for medical students and postgraduate trainees. Publication and research experience before completion of medical school are important correlates

Received September 3, 2008; accepted September 17, 2008.

Correspondence: Charles V. Smith, Ph.D., Center for Developmental Therapeutics, Seattle Children's Hospital Research Institute, 1900 Ninth Ave, Seattle, WA 98101; e-mail: charles.smith@seattlechildrens.org

Supported by APS-SPR, HD007446, individual institutions, and investigators.

Supplemental digital content is available for this article. Direct URL citations appear in the printed text and are provided in the HTML and PDF versions of this article on the journal's Web site (www.pedresearch.org). to subsequent academic careers (8), and publication before completion of medical school was the strongest correlate with entrance into academic careers.

The American Pediatric Society (APS)-Society for Pediatric Research (SPR) Medical Student Research Program (MSRP) was initiated in 1991 and designed to address the challenges in encouraging medical students to pursue careers in academic medicine. The MSRP accepts applications from medical students, typically in their first or second years of medical school, and offers 8-12 wk of support for training in research laboratories not associated with the applicants' medical schools. Funds are provided to help defray expenses of living in a different city. Participation by underrepresented minority students is encouraged, and such students are recruited actively.

The present report is not the result of a blinded, randomized trial of the effects of participation, but represents an effort to assess the impact of the MSRP, using the most objective approaches and criteria available.

\section{METHODS}

In 2001, we evaluated all of the applicants to the MSRP from 1991 through 2000. The applicants' data on year of application, participation, gender, self-identified ethnicity, medical school attended, and applicant's lab supervisor were obtained from the database maintained by APS-SPR. During 1991-2000, 28 applicants who were offered support by the MSRP chose not to participate, and three applicants who were not selected for support were funded by the respective lab in which the student worked. Gender was requested for applicants after 1992. For analysis of data from applicants in 1991 and 1992, gender was assigned for reasonably gender-specific names but was left unassigned for names not regarded as sufficiently gender specific. Self-identified race or ethnicity was requested for applicants from 1993 forward. Applicants from 1991 or 1992, applicants who did not participate in 1993, and the 28 applicants from 1994 to 2000 who did not self-identify with a racial or ethnic group were entered as None Listed. Data from the two applicants who identified themselves with two different race/ethnic groups were included with both groups.

Evidence for publications was sought for each applicant, using PubMed and a systematic strategy with the student's name and sequential Boolean refinements, using the applicant's medical school, country, MSRP mentor's name and institution, and the state of the applicant's medical school, in addition to other relevant information were obtained from the APS-SPR database. The manner in which these parameters were used in search refinement is provided in supplementary material available

Abbreviations: APS, American Pediatric Society; MSRP, Medical Student Research Program; SPR, Society for Pediatric Research 
online at www.pedresearch.org (see Supplemental Digital Content 1, http://links.lww.com/A958).

MSRP participants during 1991 through 1996 were assessed similarly in 2008, using the systematic PubMed strategy described above. Participants during this period also were investigated by combined PubMed and Google searches, to identify present professional positions, which were categorized as pediatric or nonpediatric and academic or nonacademic. Nonparticipant applicants were not examined in these more recent assessments.

Nominal data were assessed statistically by $\chi^{2}$ tests. Parametric data were assessed with independent samples $t$ tests, one-way analysis of variance with Student-Newman-Keuls tests post hoc, or with linear regression analyses, with differences noted at $p<0.05$.

\section{RESULTS}

Of the applicants who chose to self-identify with AfricanAmerican, Asian, Caucasian, or Hispanic races or ethnicities, the participation percentages over the period from 1991 to 2000 were between 31 and $36 \%$, evidencing uniform participation ( $p=0.283$ by $\chi^{2}$ analysis) across these four racial or ethnic groups (Table 1). Pacific Islanders' participation of $60 \%$ was associated with small numbers of applicants $(n=$ 15) during this period. The Pacific Islander ethnic group was not listed in the forms until 1997. At the time of the 2001 comparisons, no applicants had self-identified as Native American, even though this option had been included since 1993. For the 249 applicants identified in the 2001 data as None Listed, 220 (88.4\%) were from 1991 to 1993, during which time race/ethnicity identification was not requested from the participants (1991-1992) or from the nonparticipants (1991-1993). The additional 29 individuals included in the None Listed category from 1994 to 2000 either declined to self-identify as a member of a racial or ethnic group or may have overlooked this part of the application.

The 2001 cross-sectional comparison showed that the percentages of applicants for whom PubMed searches provided evidence of subsequent publication were higher for the MSRP participants than for nonparticipants (Fig. 1A). The percentages of former applicants who had published at least one PubMed-retrievable article increased with time for the participants $(p<0.001)$ but not for the nonparticipant applicants $(p=0.182)$

African-American, Asian, Caucasian, and Hispanic participants were more likely to have published one or more articles than were nonparticipants of the same racial or ethnic group

Table 1. MSRP participation self-identified races and ethnicities, 1991-2000

\begin{tabular}{lccc}
\hline \multicolumn{1}{c}{ Race/ethnicity } & Applicants & Participants & Participation $(\%)$ \\
\hline African-American & 128 & 45 & 35.2 \\
Asian & 335 & 114 & 33.9 \\
Caucasian & 391 & 123 & 31.3 \\
Hispanic & 66 & 24 & 36.4 \\
Pacific Islander & 15 & 9 & 60.0 \\
None listed & 249 & 50 & 20.0 \\
Total & 1184 & 365 & 30.8 \\
\hline
\end{tabular}

Self-identified race or ethnicity was not requested from applicants in 1991 or 1992, or from applicants in 1993 who did not participate; in addition, 29 applicants from 1994 to 2000 did not self-identify with a racial or ethnic group. These students are entered as None Listed. Data from two applicants who identified with two different race/ethnic groups are included with both groups. Participation rates among racial/ethnic groups were not different $(p=$ 0.283 ) by $\chi^{2}$ analyses.
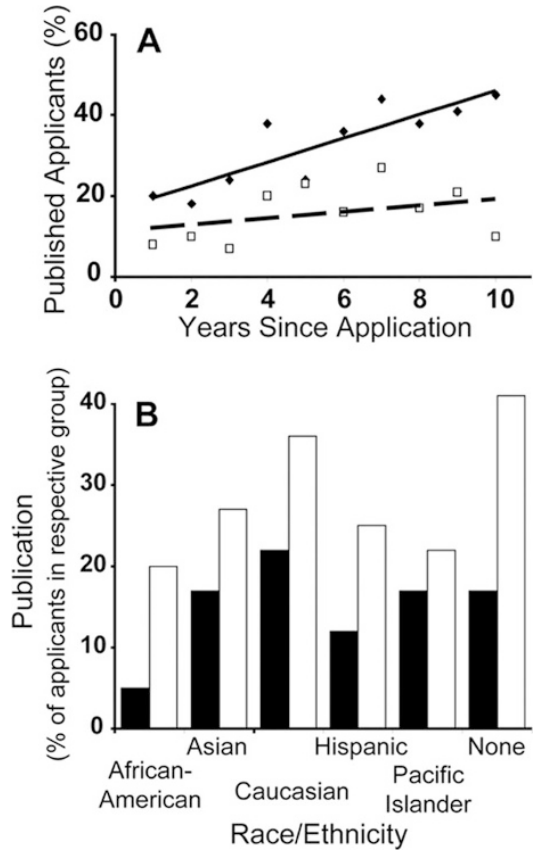

Fig. 1. Applicant publication rates, as related to years since application to the MSRP and race or ethnicity. Searches were conducted spanning the year of application through the time of the PubMed search, in 2001. A, Participants $(\diamond)$, solid line $(y=2.9455 x+16.6 ; p<0.001 v s$ zero slope); nonparticipants $(\square)$, dashed line $(y=0.9636 x+10.6) ; p=0.182$ vs zero slope. $B$, Percents of applicants with at least one publication, as distinguished by self-identified ethnic groups. (ם) Nonparticipants; ( $\square$ ) participants.

( $p=0.033,0.003,0.003$, and 0.008 , respectively), with ratios ranging from 1.6 for Asians to 4.0 for African-Americans (Fig. 1B). Among students with no race or ethnicity identified, the publication rates by participants and nonparticipants were not different $(p=0.075)$. The publication percents of Pacific Islanders were not different $(p=0.925)$, but this comparison was based on small sample sizes ( 9 and 6 , respectively).

Overall, MSRP participants were $79 \%$ more likely to have published than were nonparticipants $(p<0.0005)$. Female participants were twice as likely to have published (Fig. 2A), as were female nonparticipants $(p<0.0005)$, whereas male MSRP participants were associated with a $65 \%$ greater probability of subsequent publication than male nonparticipants $(p=0.008)$. The publication percents for male and female nonparticipants were not different $(p=0.090)$ nor were publication percents between male and female participants $(p=0.956)$.

The numbers of publications per person were higher $(p=$ 0.007 ) for participants than for nonparticipants (Fig. 2B). The average numbers of publications for female participants were greater $(p<0.0005)$ than for female nonparticipants, whereas the same comparison between participant and nonparticipant males does not indicate differences $(p=0.089)$. Comparisons between males and females, among participant and nonparticipant applicants, indicate greater numbers of publications per applicant by nonparticipant males than by nonparticipant females $(p=0.004)$, whereas a similar gender disparity is not observed among participants $(p=0.196)$.

Although MSRP participation was not associated with differences $(p=0.069)$ in the probability that a student would 

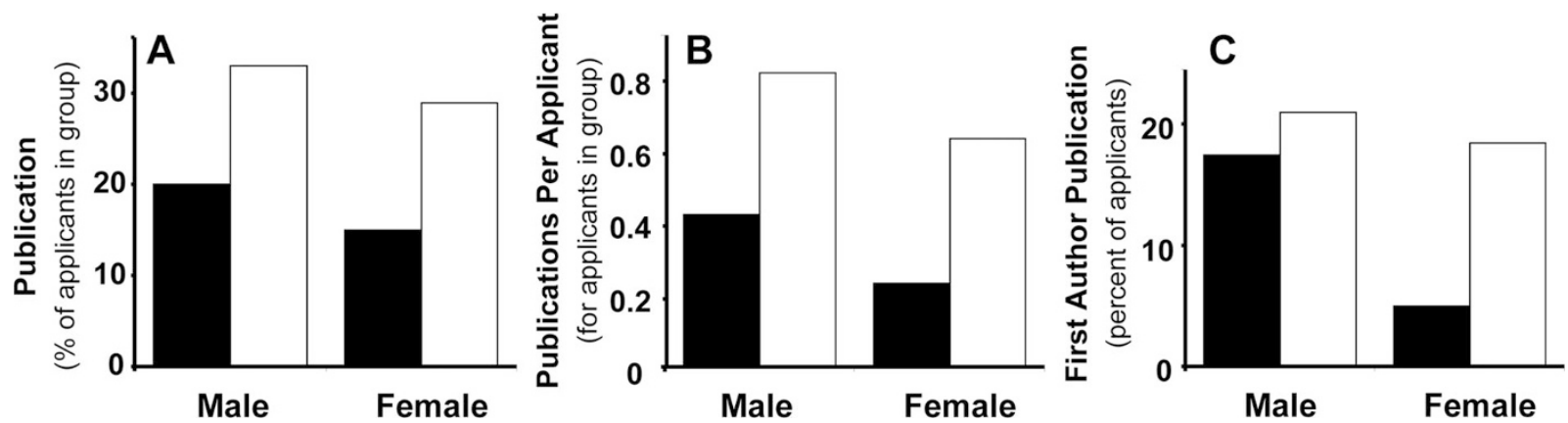

Fig. 2. Publication rates of applicants by gender. Participants ( $\square$; 146 males and 217 females) and nonparticipants ( $\square$; 325 males and 471 females). A, The percent of applicants with PubMed-identifiable publications, distinguished by gender and MSRP participation. $B$, Publications per applicant. One male participant had 71 publications ( 31 as first author) at the time of the initial analysis. Data on this person were omitted as an outlier from analysis for $(B)$. As of 2008 , this former student has 151 publications listed. $C$, Percent of applicants with one or more first author publications, distinguished by gender and MSRP participation.

publish one or more first author papers (Fig. 2C), a difference between all male and female applicants is indicated $(p=$ 0.033). This difference in percentage of applicants who subsequently published at least one first author paper is driven by the gender difference in first author publications by the nonparticipants, with male nonparticipants over three times as likely to have published at least one first author paper than female nonparticipants $(p<0.0005)$. This gender effect in percentages of first author publications was not observed among MSRP participants $(p=0.313)$, suggesting a unique contribution of MSRP participation to the research career development of female medical students.

By 2008, most of the students who participated in the MSRP between 1991 and 1996 had completed their training and made career decisions that would provide an informative assessment of their professional intentions and initial goals. Assessment by MSRP year revealed that between 12 and 28\% of the participants between 1991 and 1996 had published one or more articles with their respective MSRP mentors (Fig. $3 A$ ); however, the total publication rates for these students ranged between 40 and $65 \%$.

Focused, systematic search strategies provided present status data for slightly more than $94 \%$ of the students from MSRP years 1991-1996. Between 26 and $47 \%$ of the total participants in each year were in pediatrics, roughly two to three times the national averages for medical school graduates. More remarkably, from 23 to $45 \%$ of the total participants for each year were in academic pediatric positions (Fig. $3 B$ ).

\section{DISCUSSION}

The use of nonparticipant applicants as controls for the evaluation of the possible effects of the MSRP on student research career development has limitations. The most obvious limitation is the extent to which selection bias results in two groups that are not equal at the outset, as would be needed for objective assessments of the benefits of MSRP participation. The MSRP was never intended to be a randomized control trial to test hypotheses regarding the social psychology of research training. If the present results are dominated by effective selection of students that are more capable and/or more dedicated to academic careers, at least the MSRP has invested in the research training of a promising group of clinician-scientists.
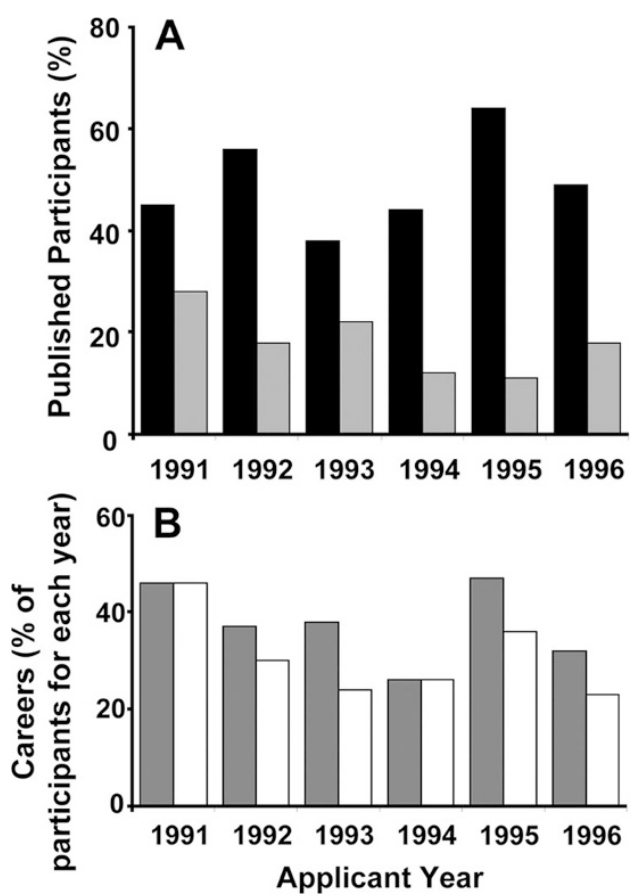

Fig. 3. Professional progression of MSRP participants between 1991 and 1996. With identification of greater than $94 \%$ of MSRP participants between 1991 and 1996 ( $n=11,31,42,34,36$, and 35, respectively). $A$, The percent of students who had published with their respective MSRP mentors ( $\square$ ), and the students for whom one or more publications were identified (ם) as of 2008. The data are normalized to total MSRP participants for each respective year. $B$, The percentages of students in pediatrics $(\square)$ and in academic pediatrics $(\square)$ are of total MSRP participants for that year.

Publication was chosen as the primary early indicator of academic career progression, because early publication is the single strongest correlate with pursuit of a career in academic medicine (8). PubMed-retrievable publications were chosen for the present evaluations, rather than questionnaire-based methods, to collect objective data and avoid potential response biases between participant and nonparticipant students. Acquisition of independent grant funding and promotion in academic rank would be stronger indicators of academic career progression by clinician-scientists, but these milestones will require longitudinal studies to collect the appropriate data.

Within the limitations provided by the methods used, the greater publication rates observed among participants than 
nonparticipants suggest that the MSRP selection process effectively identifies promising students and/or MSRP participation itself has beneficial effects. Because most students applied to the MSRP during their first year of medical school, with the research experience usually occurring during the summer between the first and second years of medical school, the increase in publication percentages over time (Fig. 1) suggests continued progression of academic career development in the MSRP participants but not in nonparticipant applicants.

The markedly greater publication rates among AfricanAmerican and Hispanic participants than among their respective nonparticipant applicants (Fig. 1B) suggest that the MSRP may be having considerable impact among underrepresented minorities in recruitment, encouragement, and training for careers as clinician-scientists. Similar successes have been observed with the Stanford Medical Youth Science Program, which focuses on encouraging and mentoring socioeconomically underprivileged high school students to enter scientifically related fields (9).

Although not an original focus of the MSRP, the unanticipated gap between male and female nonparticipants for first author publications (Fig. 2C) suggests a gender bias in academic career development that has received limited attention (10). The implications of the gender-based differences in publication rates by nonparticipants have not been established, but the differences in the percentages of male and female nonparticipants with one or more first author publications are striking. Conversely, the absence of gender-based differences in first author publications by the participants strongly suggests that the MSRP has positive effects on academic career development among female medical students. The possibility that the data on first author publications could be attributed to selection bias is highly unlikely, because this interpretation would require that the steering committee was somehow more able to distinguish capable and dedicated applicants among females than among males.

The rates of publication with their respective MSRP research mentors by the students from 1991 through 1996 are modest, ranging between 12 and 28\% for each year (Fig. $3 A$ ). Two to $3 \mathrm{mo}$ is a limited period of time for completion of a research project, even for talented and committed students in productive labs. However, the longer term publication frequencies of these students, ranging between 37 and $65 \%$ for these MSRP years, suggest substantive engagement of these students in research and academics. The choice of a career in pediatrics by 26 to $39 \%$ of these students (Fig. $3 B$ ), at two to three times the national average of $13 \%$, for graduates of US medical schools (11), is reasonably attributable to selection bias for preexisting interests in pediatrics, but contributions from exposure in the MSRP are not excluded.

Solomon et al. (12) reported that medical students formerly participating in summer research programs at the University of Tennessee College of Medicine and Vanderbilt University
School of Medicine exhibited high rates of publication and of current participation in academic medicine. However, the data reported by Solomon et al. were based on questionnaire replies, with response rates in the range of $30 \%$, and selection bias in responses by the former students would not be unexpected.

The vast majority of the former MSRP students who are in pediatrics are in academic pediatric positions (Fig. $3 B$ ), which is most remarkable. The data presented in Figure $3 B$ for students from 1991 to 1996 in academic pediatric positions in 2008 are not percentages of students in pediatrics but of all students who participated in the MSRP in the years indicated. The data represent rates of entry into academic pediatrics that are roughly 10 times the national averages for US medical school graduates (1).

The MSRP clearly has been successful in attracting large numbers of highly qualified medical student applicants, particularly among women and members of underrepresented racial and ethnic groups. The higher rates of publication by participants than by nonparticipant applicants suggest that the selection process has been effective in identifying the more promising students and/or MSRP participation has contributed to the career development of the students. The most encouraging data supporting the contributions of this MSRP to the stated goal of recruitment and training of pediatric clinicianinvestigators are the remarkable numbers of former students who participated in the MSRP from 1991 through 1996 who have entered careers in academic pediatrics.

Acknowledgments. Special thanks to Ms. Barbara Anagnostelis, whose tireless efforts in managing the many details essential to the operation of the program have been deeply appreciated by all those involved.

\section{REFERENCES}

1. Jobe AH, Abramson JS, Batshaw M, Boxer LA, Lister G, McCabe E, Johnston R 2002 Work Groups on Research, American Pediatric Society Recruitment and development of academic pediatricians: departmental commitments to promote success. Pediatr Res 51:662-664

2. Shaul PW 2007 The cold, hard truth about pediatric research. Pediatr Res 61:506-509

3. Ley TJ, Rosenberg LE 2005 The physician-scientist career pipeline in: build it, and they will come. JAMA 294:1343-1351

4. Betancourt JR, King RK 2000 Diversity in health care: expanding our perspectives. Arch Pediatr Adolesc Med 154:871-872

5. Cohen JJ 2003 The consequences of premature abandonment of affirmative action in medical school admissions. JAMA 289:1143-1149

6. Komaromy M, Grumbach K, Drake M, Vranizan K, Lurie N, Keane D, Bindman AB 1996 The role of black and Hispanic physicians in providing health care for underserved populations. N Engl J Med 334:1305-1310

7. Rabinowitz HK, Diamond JJ, Veloski JJ, Gayle JA 2000 The impact of multiple predictors on generalist physicians' care of underserved populations. Am J Public Health 90:1225-1228

8. Ledley FD, Lovejoy FH Jr 1992 Prospects for academically trained pediatricians in academic medicine. Clin Invest Med 15:518-526

9. Winkleby MA 2007 The Stanford Medical Youth Science Program: 18 years of a biomedical program for low-income high school students. Acad Med 82:139-145

10. Jagsi R, Guancial EA, Worobey CC, Henault LE, Chang Y, Starr R, Tarbell NJ, Hylek EM 2006 The "gender gap" in authorship of academic medical literature-a 35-year perspective. N Engl J Med 355:281-287

11. Newton DA, Grayson MS 2003 Trends in career choice by US medical school graduates. JAMA 290:1179-1182

12. Solomon SS, Tom SC, Pichert J, Wasserman D, Powers AC 2003 Impact of medica student research in the development of physician-scientists. J Investig Med 51:149-156 